\title{
Artículo
}

\section{Lipodistrofia tipo Köbberling, una enfermedad subdiagnosticada}

\section{Köbberling type Lipodystrophy, an underdiagnosed disease.}

Autores

Dr. Daniel Chaverri Guilén (receptor de correspondencia)

Investigador independiente, San José, Costa Rica

Dr. Alejando Cob Guillén

Endocrinólogo CCSS, San José, Costa Rica

Dr.José/Roberto Chacón Barrantes

Investigador independiente, San José, Costa Rica jose2505cr@gmail.com

Correspondencia: José Roberto Chacón Barrantes Correo electrónico: jose2505cr@gmail.com

\section{Resumen}

La lipodistrofia es una enfermedad en la que se asocia tanto pérdida como redistribución y acúmulo del tejido adiposo, estos cambios conociéndose como lipoatrofia y lipohipertrofia, respectivamente. Estas alteraciones del tejido adiposo llevan a deficiencia de hormonas de origen adipocitario, causando anomalías metabólicas y depósitos de adipocitos en diferentes órganos diana. Las lipodistrofias se pueden diferenciar dependiendo del mecanismo de adquisición, siendo congénitas (familiares) o adquiridas, y por la distribución o pérdida de grasa, en parciales o generalizadas. En este artículo se relata el caso clínico de una paciente con Lipodistrofia parcial familiar tipo 1 , o de Köbberling, la cual se diferencia de los otros tipos por no presentar alteración genética conocida, por la disminución de tejido adiposo a nivel de extremidades y el aumento de este mismo en tronco, cuello y cara. 
Se ha definido como una enfermedad subdiagnosticada, aunque al sospechar de ella se pueden emplear diversos estudios de imagen y de laboratorio para su diagnóstico. No hay un tratamiento establecido para esta patología, y el manejo recae en el control de las alteraciones metabólicas.

\begin{abstract}
Lipodystrophy is a disease in which both, loss or/and redistribution plus gain of adipose tissue are associated, these changes being known as lipoatrophy and lipohypertrophy, respectively. These adipose tissue alterations lead to deficiency of fat tissue hormones, causing metabolic abnormalities and adipocyte deposits in different organs. Lipodystrophies can be differentiated depending on the mechanism of acquisition, being congenital (familial) or acquired, and depending on the distribution or loss of fat, in partial or generalized. This article relates the clinical case of a patient with familial partial lipodystrophy type 1 , or Köbberling type, which differs from the other types by not having a known genetic alteration, but also by the decrease of adipose tissue at the extremities and the increase of the same in the trunk, neck and face. It has been defined as an underdiagnosed disease, although a number of laboratory and imaging studies may be used upon suspicious of it. There is no established treatment for this pathology, and the manage remains in the control of the metabolic alterations.
\end{abstract}

\section{Palabras clave}

Lipodistrofia; dislipidemia; diabetes; ateroesclerosis; leptina; genética

\section{Introducción}

Las lipodistrofias son un grupo de patologías poco prevalentes y raras de observar en la consulta diaria de un médico. En 1911, Simons describe el caso de una mujer joven, que a los 11 años desarrolló pérdida de tejido adiposo a nivel de cara, extremidades y tronco, usando el término Lipodistrofia (palabra griega que se refiere a "trastorno del metabolismo de las grasas") 1 .

Las lipodistrofias se caracterizan por ser desordenes heterogéneos, autosómicos y con variedad de fenotipos2. La diferenciación de cada síndrome es complicada debido a las similitudes antropométricas, clínicas y metabólicas3. Etiológicamente se ha demostrado que esta patología se presenta mayoritariamente en la infancia y en mujeres, donde se pueden presentar síntomas de síndrome de ovario poliquístico4 y en las cuales hay que diferenciar de un cuadro clínico clásico de enfermedad de Cushing. Normalmente se presentan como un déficit parcial o generalizado de tejido adiposo a nivel corporal, conocido como lipoatrofia5, estableciéndose por carencias nutricionales o estados hipercatabólicos. Este déficit puede verse acompañado por la rara redistribución de tejido adiposo a diferentes lugares del cuerpo humano, lo que se conoce como lipohipertrofia. La reducción en tejido adiposo puede verse reflejado a nivel hormonal como una disminución o ausencia de hormonas adipoquinas, que se encargan de la regulación del metabolismo glucídico, lipídico y energético. La principal hormona afectada es la leptina, y su disminución se encuentra vinculada con la hiperfagia y la resistencia a insulina mostrada en estos pacientes. Esta hormona tiene efectos importantes sobre el apetito, el balance energético y está asociada al funcionamiento neuroendocrinológico e inmunológico6. Los niveles séricos de esta hormona son directamente proporcionales con la cantidad de masa adipocitaria7 presente en el cuerpo humano.

La lipodistrofia no solo va a afectar el tejido adiposo subcutáneo, sino que también el tejido visceral, por migración adipocitaria. Entre las alteraciones que puede causar se encuentran la resistencia a la insulina, la hiperglicemia, las dislipidemias importantes (especialmente hipertrigliceridemias), el daño hepático 
progresivo y un aumento de gasto metabólico. Vamos a diferenciar las lipodistrofias dependiendo del mecanismo de adquisición y lugar de afectación, siendo estas congénitas (familiares) o adquiridas, y como generalizadas o parciales. Esta enfermedad va a depender del desorden autosómico que presente, siendo en las parciales dominante y en las generalizadas recesivo. La lipodistrofia más frecuente es a causa de tratamiento antirretroviral en paciente con VIH, siendo esta un efecto adverso común8. Este artículo trata de una paciente con un tipo de lipodistrofia parcial, la cual es subdiagnosticada por los médicos, debido a la poca sospecha de estos sobre esta patología.

\section{Caso Clínico}

Paciente femenina de 39 años, vecina de Limón, con antecedente de un rápido aumento de peso a partir de los 20 años, llegando hasta un peso máximo de $87 \mathrm{~kg}$ (IMC $37.8 \mathrm{~kg} / \mathrm{m} 2$ ) actualmente. Con el aumento de peso, la paciente inició con múltiples episodios a repetición de pancreatitis aguda, asociada a hipertrigliceridemia, cada vez más severas requiriendo múltiples internamientos en la Unidad de Cuidados Intensivos, con necesidad de plasmaféresis. La paciente fue referida a endocrinología del Hospital Tony Facio Castro para control de sus comorbilidades metabólicas. Padece dislipidemia mixta documentada a los 27 años, diabetes mellitus tipo 2 diagnosticada a los 35 años, no es hipertensa, ni tabaquista.

En la valoración por endocrinología, se evidencia una paciente con obesidad central, aumento de tejido adiposo supraclavicular y retrocervical, sin estrías, rubor facial o hirsutismo. Llamó la atención la disminución de tejido subcutáneo en las extremidades. Los brazos son delgados, se logra visualizar sistema venoso superficial y hay un claro límite donde se define la disminución de la grasa subcutánea a nivel del tercio proximal de ambos brazos. Las piernas también son delgadas, se le logran definir los músculos, y de la misma forma, hay una clara división donde se delimita la disminución de tejido graso subcutáneo a nivel de los glúteos (Figura 1).

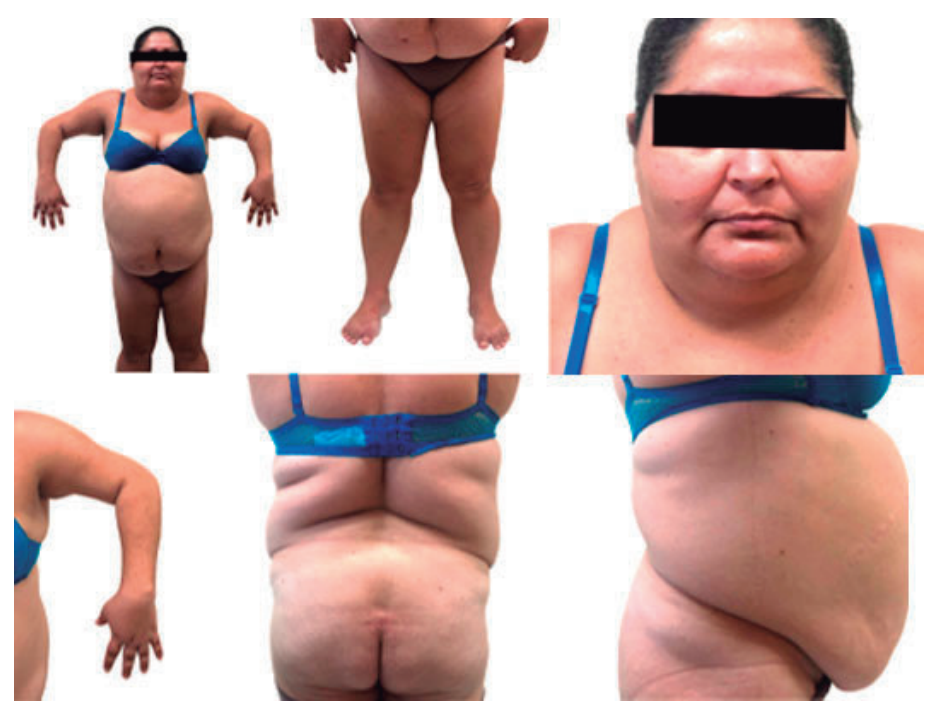

Figura 1: Características clínicas de lipodistrofia de Köbberling. A) obesidad central con extremidades delgadas. B) se visualiza la musculatura de las piernas. C) Facies luna llena, adiposidad supracalvicular D) se evidencia límite de tejido adiposo a nivel triceps con flecha. E y F) se evidencia limite de tejido adiposo sobre glúteo con flecha.

Adicionalmente presentó amenorrea de 1 año de evolución y durante el seguimiento médico se descartó Síndrome de Cushing, hiperandrogenemia y disfunción tiroidea corporal mediante una balanza con impedanciometría, documentan peso $87 \mathrm{~kg}$, con IMC $37.8 \mathrm{~kg} / \mathrm{m} 2$ y porcentaje de grasa corporal de $46.2 \%$. Monitoreo de glucosa contínuo por 7 días, que muestra un tiempo en rango de $16 \%(70-180 \mathrm{mg} / \mathrm{dl})$, sin hipoglicemias, consumiendo un promedio de 135gr de carbohidratos por día.

Actualmente la paciente recibe tratamiento con, Fenofibrato $250 \mathrm{mg} /$ día, Rosuvastatina $40 \mathrm{mg} /$ día, $\mathrm{y}$ un esquema intensificado de Insulina a $2 \mathrm{U} / \mathrm{kg} /$ día.

Su control metabólico en la última visita médica presentó mejoría desde el último episodio de pancreatitis, aunque persiste fuera de metas pese a tratamiento y educación en nutrición 
(Tabla 1).

Ante la sospecha clínica se le realiza una medición de leptina, con resultado de $10.4 \mathrm{ng} / \mathrm{ml}$, siendo esto desproporcional a su composición corporal9. pacientes presentan cuadros de hipertensión arterial, acantosis nígricans, esteatosis hepática, diabetes mellitus e hipertrigliceridemias, llegando a causar daños cardiovasculares, hepáticos y pancreáticos, irreversibles 12 .

\begin{tabular}{|c|c|c|c|}
\hline Fecha & Julio 2018 & Octubre 2018 & Enero 2018 \\
\hline Hba1c & Julio 2018 & $8.3 \%$ & $7.5 \%$ \\
\hline Triglicéridos & $4886 \mathrm{mg} / \mathrm{dl}$ & $2403 \mathrm{mg} / \mathrm{dl}$ & $1036 \mathrm{mg} / \mathrm{dl}$ \\
\hline Colesterol HDL & $8 \mathrm{mg} / \mathrm{dl}$ & $18 \mathrm{mg} / \mathrm{dl}$ & $18 \mathrm{mg} / \mathrm{dl}$ \\
\hline Colesterol no HDL & $556 \mathrm{mg} / \mathrm{dl}$ & $252 \mathrm{mg} / \mathrm{dl}$ & $133 \mathrm{mg} / \mathrm{dl}$ \\
\hline AST/ALT & $432 / 435 \mathrm{UI} / \mathrm{L}$ & 54/44 IU/L & $33 / 28 \mathrm{IU} / \mathrm{L}$ \\
\hline
\end{tabular}

Tabla 1: Control metabólico de la paciente

\section{Discusión}

Existen varios tipos de lipodistrofias parciales familiares, estas se distinguen por una causa genética diferente. La lipodistrofia de tipo Köbberling es infrecuente y no se ha podido concretar una mutación genéticaespecífica10 que presenta, a diferencia de los otros síndromes que presentan una mutación conocida en el gen de lamina LMNA A/C o PPAR. Entre estas se conoce que la lipodistrofia parcial familiar tipo 2, de Dunnigan, es la más común.

La lipodistrofia tipo Köbberling normalmente se caracteriza por una lipoatrofia de tejido subcutáneo en extremidades11 y una lipohipertrofia en cuello, cara y tronco. Se presenta con afectación orgánica moderada/grave al momento del diagnóstico, debido a la poca frecuencia y a la dificultad para diferenciar esta enfermedad de un síndrome metabólico. Los
Entre las afecciones más importantes se presenta la enfermedad coronaria temprana, a causa de la ateroesclerosis que puede ocasionar por la gran cantidad de lípidos en torrente sanguíneo y su alto índice de mortalidad por daño miocárdico13. Es claro que el fenotipo de la enfermedad es más prominente cuando el paciente gana peso y empeora en las mujeres durante la menopausia14.

El diagnóstico es complicado, al no contar con una mutación genética específica, se debe basar en las características físicas de cada persona y en las alteraciones metabólicas que presente. Una vez sospechada la enfermedad se puede cuantificar a nivel sérico la leptina, siendo la principal hormona afectada en está patología. La leptina se sintetiza y se secreta mayoritariamente por el tejido

adiposo, aunque también en otros tejidos como 
la glándula mamaria, los ovarios, la placenta, el estómago, los testículos, la hipófisis, el hipotálamo, el endometrio, entre otros. Las mujeres presentan mayor concentración de leptina que los hombres, porque tienen mayor cantidad de tejido adiposo. Los niveles en sangre de esta hormona se presentan directamente proporcional a la cantidad de grasa del cuerpo, por lo que se encuentran alterados en casos de lipodistrofia. Se puede diferenciar esta patología con otros tipos de lipodistrofia al realizar estudios genéticos basados en dos genes responsables, la LMNA y el PPARG; lastimosamente el test es positivo en apenas un $50 \%$ de los casos, por lo que el diagnóstico se basa en el fenotipo del paciente15. Los estudios de imagen ayudan al diagnostico preciso y comparativo de cada paciente, entre estos tenemos: la resonancia magnética como Gold-Standard, la tomografía axial computarizada y la absorciometría de energía dual de rayos X (DEXA), siendo esta última utilizada para estimar el porcentaje de masa grasa, masa magra y masa ósea. Debido al alto costo e irradiación de los procedimientos la recomendación ha sido estandarizar el diagnóstico al presentar en el DEXA una pérdida del tejido adiposo periférico en un porcentaje mayor al $20 \%$, y/o un aumento mayor a un $20 \%$, concomitante con alteraciones en los resultados de laboratorio.

Por el momento no existe ningún tratamiento etiológico para la lipodistrofia familiar parcial, sin embargo, en las lipodistrofias generalizadas se ha empleado un análogo de leptina humana recombinante de vida media-larga, llamado Metroleptina16, que se utiliza como reemplazo para el déficit de esta hormona. Se ha demostrado que el uso de la leptina revierte tanto la resistencia a insulina por el músculo y el hígado, como la esteatosis hepática17. Regularmente los pacientes con lipodistrofia requieren tratamiento antidiabético e hipolipemiantes, siendo útiles únicamente para las anormalidades metabólicas18. Otra medida terapéutica utilizada es la cirugía bariátrica19, que ha sido de gran ayuda para estos pacientes tanto metabólicamente como en la parte estética, siendo importante está última para la salud mental de cada individuo.

\section{Conclusión}

Las lipodistrofias son patologías mas frecuentes de lo que se consideraba previamente, la mayoría de estas pasan subdiagnosticadas por la falta de sospecha clínica de los médicos. Su diagnostico temprano es de gran importancia para disminuir las alteraciones metabólicas. La afectación al metabolismo del tejido adiposo causa grave resistencia a insulina y un desbalance glicémico marcado.

En algunas condiciones específicas, la terapia de reemplazo de Leptina ha demostrado mejorar el control glicémico, la hipertrigliceridemia, y la esteatosis hepática no alcohólica, sin embargo, es importante tratar las alteraciones metabólicas como la dislipidemia, la hiperinsulinemia y el déficit nutricional con tratamiento para estos. Se debe profundizar en la salud mental del paciente, ya que existe gran afectación a nivel psicológico por sus cambios físicos, causando indisposición de estas personas a llevar una vida social con normalidad y asistir a sus citas médicas.

Al ser una enfermedad hereditaria y de expresión variable, es importante vigilar a los familiares de estos pacientes, como en este caso que nuestra paciente tiene una hija de 20 años, la cual ya está adoptando un fenotipo similar al de su madre, aún sin presentar trastornos metabólicos y hormonales.

\section{Referencias}

1. Misra, A., Peethambaram, A., \& Garg, A. Clinical Features and Metabolic and Autoimmune Derangements in Acquired Partial Lipodystrophy. Medicine, 2004, 83(1), 18-34. 
2. Garg, A. Acquired and Inherited Lipodystrophies. New England Journal of Medicine, 2004 March 18,350(12), 1220-1234.

3. Guillín-Amarelle, C., Sánchez-Iglesias, S., Castro-Pais, A., Rodriguez-Cañete, L., Ordóñez-Mayán, L., Pazos, M., ... Araújo-Vilar, D. Type 1 familial partial lipodystrophy: understanding the Köbberling syndrome. Endocrine, 2016 May 26, 54(2), 411-421.

4. Garg, A., \& Agarwal, A. K. Lipodystrophies: Disorders of adipose tissue biology. Biochimica et Biophysica Acta (BBA) Molecular and Cell Biology of Lipids, 2009, 1791(6), 507-513.

5. Garg, A. Lipodystrophies: Genetic and Acquired Body Fat Disorders. The Journal of Clinical Endocrinology \& Metabolism, 2011 Nov, 96(11), 3313-3325.

6. Ahima, R. S., \& Flier, J. S. Adipose Tissue as an Endocrine Organ. Trends in Endocrinology \& Metabolism, 2002, 11(8), 327-332.

7. Oral, E. A., Simha, V., Ruiz, E., Andewelt, A., Premkumar, A., Snell, P., ... Garg, A. (2002). Leptin-Replacement Therapy for Lipodystrophy. New England Journal of Medicine, 2002, 346(8), 570-578.

8. Spuler, S., Kalbhenn, T., Zabojszcza, J., van Landeghem, F. K. H., Ludtke, A., Wenzel, K., ... Schmidt, H. H. Muscle and nerve pathology in Dunnigan familial partial lipodystrophy. Neurology, 2007, 68(9), 677-683.

9. Faisal, R., Hassan, M., Nazar, HS., Gillani, S., Afzal, N., Gayyum, I. Effect of body mass index on serum leptin levels. Journal of Ayub Medical College, Abbottabad, 2011 December.
10. Lightbourne, M., \& Brown, R. J. Genetics of Lipodystrophy. Endocrinology and Metabolism Clinics of North America, 2017,46(2), 539-554.

11. Herbst, K. L., Tannock, L. R., Deeb, S. S., Purnell, J. Q., Brunzell, J. D., \& Chait, A. Kobberling Type of Familial Partial Lipodystrophy: An underrecognized syndrome. Diabetes Care, 2003 June, 26(6), 1819-1824.

12. Akinci, B., Onay, H., Demir, T., Savas-Erdeve, Ş., Gen, R., Simsir, I. Y.Oral, E. A. Clinical presentations, metabolic abnormalities and end-organ complications in patients with familial partial lipodystrophy. Metabolism, 2017, 72, 109-119.

13. McLaughlin, P. D., Ryan, J., Hodnett, P. A., O'Halloran, D., \& Maher, M. M. Quantitative Whole-Body MRI in Familial Partial Lipodystrophy Type 2: Changes in Adipose Tissue Distribution Coincide With Biochemical Improvement. American Journal of Roentgenology, 2012, 199(5), W602-W606.

14. Aberer F, Sourij H, Mader JK. A typical clinical presentation of a woman with Köbberling syndrome. Pol Arch Intern Med. 2019; 129: 414-416.

15. Joy, T., Kennedy, B. A., Al-Attar, S., Rutt, B. K., \& Hegele, R. A. Predicting abdominal adipose tissue among women with familial partial lipodystrophy. Metabolism, 2009, 58(6), 828-834.

16. Chou, K., \& Perry, C. M. Metreleptin: First Global Approval. Drugs, 2013 June 6, 73(9), 989-997.

17. Petersen, K. F., Oral, E. A., Dufour, S., Befroy, D., Ariyan, C., Yu, C., ... Shulman, G. I. Leptin reverses insulin resistance and hepatic steatosis in patients with severe lipodystrophy. Journal of Clinical Investigation, 2002, 109(10), 1345-1350. 
18. Chan, J., Lutz, K., Cochran, E., Huang, W., Peters, Y., Weyer, C., \& Gorden, P. Clinical Effects of Long-Term Metreleptin Treatment in Patients with Lipodystrophy. Endocrine Practice, 2011, 17(6), 922-932.

19. Melvin, A., Adams, C., Flanagan, C., Gaff, L., Gratton, B., Gribble, F., ... Savage, D. B. Roux-en-Y Gastric Bypass Surgery in the Management of Familial Partial Lipodystrophy Type 1 . The Journal of Clinical Endocrinology \& Metabolism, 2017 October, 102(10), 3616-3620. 